\title{
Evaluación del método del tubo para concentrar plaquetas felinas: estudio celular
}

\author{
Evaluation of the tube method for concentrating feline platelets: cellular study \\ RF Silva $^{a, b^{*}}$, CMF Rezende ${ }^{\mathrm{b}}$, FO Paes-Leme ${ }^{\mathrm{b}}$, JU Carmona ${ }^{\mathrm{a}}$ \\ ${ }^{a}$ Grupo de Investigación Terapia Regenerativa, Departamento de Salud Animal, Universidad de Caldas, \\ Manizales, Caldas, Colombia. \\ ${ }^{\text {b} E s c o l a ~ d e ~ V e t e r i n a ́ r i a, ~ U n i v e r s i d a d e ~ F e d e r a l ~ d e ~ M i n a s ~ G e r a i s, ~ B e l o ~ H o r i z o n t e, ~ M i n a s ~ G e r a i s, ~ B r a s i l . ~}$
}

\begin{abstract}
SUMMARY
The aim of this study was to evaluate a manual tube method for concentrating cat platelets and consequently produce autologous platelet concentrates (APCs) or platelet rich plasma (PRP), for clinical and experimental purposes. The PRP were obtained by venous blood collection in tubes with ACD solution that were spun at $85 \mathrm{~g}$ for 6 minutes. The cell counts of whole blood and PRP, were statistically different $(\mathrm{P}<0.01)$ between cell values for platelets, leukocytes, granulocytes, monocytes, but not for lymphocytes. We found a strong positive correlation $(\rho=0,734 \mathrm{P}=0,01)$ between the number of platelets in whole blood and platelets in the PRP. The collection efficiency of platelets was $50 \%$ and the concentration of platelets was $147.7 \%$ higher in PRP in comparison with the whole blood samples. The results indicated that this simple centrifugation tube method allows concentrating cat platelets.
\end{abstract}

Palabras clave: gato, plasma rico en plaquetas autólogo, medicina regenerativa, método del tubo.

Key words: cat, autologous platelet rich plasma, regenerative medicine, tube method.

\section{INTRODUCCIÓN}

En condiciones fisiológicas, las plaquetas (luego de ser activadas) liberan cantidades sustanciales de factores de crecimiento (GFs), entre los que se destacan el GF transformante beta I (TGF- $\beta_{1}$ ), GF derivado de las plaquetas (PDGF) y otras moléculas que modulan la inflamación y la reparación tisular (Anitua y col 2004, Argüelles y col 2006). Debido a esta razón biológica, se ha diseminado el uso de concentrados autólogos de plaquetas (APCs) en medicina y cirugía humana en procedimientos quirúrgicos maxilofaciales (Thor y col 2007), plásticos, ortopédicos, gastroenterológicos e incluso oftalmológicos (Anitua y col 2007).

El uso clínico de los concentrados autólogos de plaquetas (APCs), también conocidos genéricamente como plasma rico en plaquetas (PRP) en medicina veterinaria, se ha focalizado en el campo de la medicina y cirugía equina. Actualmente existen algunas evidencias clínicas que sugieren efectos benéficos de esas sustancias cuando son aplicadas en caballos con afecciones locomotoras crónicas (Carmona y col 2009a,b, Carmona y Prades 2009) o con heridas de las extremidades (Monteiro y col 2009). Los APCs o el PRP han sido evaluados como coadyuvantes (únicos o combinados con biomateriales) en modelos caninos de regeneración ósea maxilar (Moreno y col 2004, Bonomi y col 2007, Dutra y col 2008) o apendicular (You y col 2007) y en pruebas de osteointegración (Casati y col 2007). Por otra parte, existe vaga documentación sobre el uso y efecto clínico de un APC en un caso de artrodesis del tarso en un perro (Hauschild y col 2005).

Los felinos pueden desarrollar diversos problemas crónicos del aparato locomotor y sufrir heridas (Chandler y Beale 2002) que pueden ser susceptibles de ser tratadas con concentrados de plaquetas. También esas sustancias podrían ser empleadas como biomateriales coadyuvantes en cirugía ortopédica (Intini 2009) en esa especie. Sin embargo, según la literatura revisada, los autores no encontraron reportes sobre protocolos de obtención de APCs o PRP en gatos con fines terapéuticos. El objetivo de la presente investigación fue estandarizar un método manual que permitiera concentrar plaquetas felinas de manera reproducible para poder usar los concentrados de plaquetas (o PRP) en el campo clínico o experimental en esa especie. La hipótesis del estudio fue que las plaquetas de gato podrían ser concentradas mediante la recolección de sangre entera en tubos con ácido cítrico-citrato de sodiodextrosa (ACD) al ser centrifugados de manera única.

\section{MATERIAL Y MÉTODOS}

Este estudio fue aprobado por el comité de ética en experimentación animal de la Universidad Federal de Minas Gerais, Brasil, protocolo número 125/2009. 
ANIMALES

Se utilizaron doce gatos sin raza definida, seis machos y seis hembras con edades entre 12 y 36 meses, con peso corporal promedio de $3,8 \mathrm{~kg}$, clínicamente sanos al momento de la recolección de la sangre, serológicamente negativos a inmunodeficiencia viral felina (FIV) y a leucemia viral felina (FeLV).

\section{RECOLECCIÓN DE LA SANGRE Y PREPARACIÓN DEL PLASMA RICO EN PLAQUETAS}

Se realizó extracción de sangre mediante punción de la vena yugular con un catéter mariposa $21 \mathrm{G}$ (Shandong Weigao Group, China). La sangre de cada animal fue depositada en un tubo de $10 \mathrm{~mL}$ con $1,5 \mathrm{~mL}$ de solución $\mathrm{A}$ de ACD, citrato de trisodio (22 g/L), ácido cítrico ( $8 \mathrm{~g} / \mathrm{L})$ y dextrosa (24,5 g/L) (BD, New Jersey, USA). Una muestra adicional de sangre fue depositada en un tubo de $5 \mathrm{~mL}$ con $\mathrm{K}_{3}$ EDTA para realizar un hemograma. La sangre del tubo con ACD fue centrifugada (Sigma 3K30, Alemania) a $85 \mathrm{~g}$ durante 6 minutos. Posteriormente, con la ayuda de una micropipeta de volumen fijo de $1.000 \mu \mathrm{L}$ se recolectaron (aproximadamente) los primeros $100 \mu \mathrm{L}$ de la porción roja y los primeros $900 \mu \mathrm{L}$ de plasma, por debajo y por encima de la interfaz eritrocitos-plasma, respectivamente.

Las fracciones de PRP obtenidas fueron analizadas mediante hemograma automatizado por impedancia volumétrica (Abacus Junior Vet, Austria). Cada muestra fue analizada por triplicado y se tomó la media como medida muestral para los análisis estadísticos. Los parámetros hematológicos evaluados incluyeron hematocrito (PCV), recuento de plaquetas (PLT), volumen plaquetario medio (MPV), leucocitos (WBC), valores relativos y absolutos de linfocitos (LYM), monocitos (MID), neutrófilos, eosinófilos y basófilos (GRA).

\section{ANÁLISIS ESTADISTICO}

Las variables hematológicas estudiadas presentaron distribución normal según la prueba de KolmogorovSmirnov $(\mathrm{P}>0,05)$. Los valores de sangre entera y del PRP fueron comparados mediante la prueba de Student (t) para muestras pareadas. Los factores sexo y edad fueron analizados mediante un análisis de varianza (ANOVA). Todos los resultados se presentaron como media y error estándar de la media. El coeficiente de correlación de Pearson $(\rho)$ fue empleado para determinar el grado de asociación entre los parámetros evaluados en los concentrados de plaquetas (o PRP) y las muestras de sangre entera. Para estudiar la relación entre variables se realizó un análisis de regresión lineal simple. Se aceptó una diferencia estadísticamente significativa de $\mathrm{P} \leq 0,01$ para todas las pruebas. La eficiencia de colección de plaquetas se determinó mediante la fórmula de Weibrich y col (2003): (volumen de PRP $\times$ recuento de plaquetas en el
PRP / volumen de sangre entera $\times$ recuento de plaquetas en sangre entera) $\times 100$.

\section{RESULTADOS Y DISCUSIÓN}

Los recuentos celulares de la sangre entera y del PRP presentaron diferencias estadísticamente significativas respecto a los valores de PLT, WBC, PCV, valores relativos y absolutos de GRA, MID y relativos de LYM, pero no se observó lo mismo para los valores absolutos de LYM (cuadro 1). No se hallaron diferencias entre el sexo y la edad de los animales y los recuentos celulares en el análisis hematológico. Se encontró correlación positiva fuerte $(\rho=0,734 \mathrm{P}=0,01)$ entre el número de plaquetas presentes en los APCs (PLT-APC) y el número de plaquetas en sangre entera. La respectiva ecuación de regresión lineal se expresó como PLT-APC = 341.163,294 + 1,650 (PLT). La eficiencia en la recolección de plaquetas fue de $50 \%$. La concentración de plaquetas fue $147,7 \%$ superior en el PRP respecto a las muestras de sangre entera. El valor medio de MPV en el PRP fue 10.45 fentolitros (fl).

La principal motivación que obligó esta investigación fue desarrollar un protocolo de centrifugación simple en tubo para obtener APCs o PRP de gatos. Si bien los autores no encontraron investigaciones sobre obtención

Cuadro 1. Valores promedios ( \pm error estándar) de las variables hematológicas en muestras de sangre entera y concentrado de plaquetas.

Mean values ( \pm standard error) for the hematologic variables in the whole blood samples and the platelet concentrates.

\begin{tabular}{lcc}
\hline Variable & Sangre entera & Plasma rico en plaquetas \\
\hline $\begin{array}{l}\text { PLT (fragmentos } \times \\
\left.10^{3} / \mu \mathrm{L}\right)\end{array}$ & $412,33(49.59)^{\mathrm{a}}$ & $1021,50(111,55)^{\mathrm{b}}$ \\
PCV \% & $33,86(1,39)^{\mathrm{a}}$ & $2,11(0,36)^{\mathrm{b}}$ \\
WBC (células $\times$ & $10,18(0,84)^{\mathrm{a}}$ & $6,59(0,78)^{\mathrm{b}}$ \\
$\left.10^{3} / \mu \mathrm{L}\right)$ & \\
LYM (células $\times$ & $2,95(0,37)$ & $3,71(0,57)$ \\
$\left.10^{3} / \mu \mathrm{L}\right)$ & \\
LYM\% & $29,73(3,22)^{\mathrm{a}}$ & $55,80(4,53)^{\mathrm{b}}$ \\
MID (células $\times$ & $0,63(0,07)^{\mathrm{a}}$ & $0,26(0,03)^{\mathrm{b}}$ \\
$\left.10^{3} / \mu \mathrm{L}\right)$ & $6,15(0,44)^{\mathrm{a}}$ & $4,35(0,52)^{\mathrm{b}}$ \\
MID\% & $6,62(0,67)^{\mathrm{a}}$ & $2,64(0,41)^{\mathrm{b}}$ \\
GRA (células $\times$ & $64,13(3,08)^{\mathrm{a}}$ & $39,98(4,19)^{\mathrm{b}}$ \\
$\left.10^{3} / \mu \mathrm{L}\right)$ & $7,57^{\mathrm{a}}$ & $10,45^{\mathrm{b}}$ \\
GRA\% & & \\
MPV (fl) &
\end{tabular}

PLT, plaquetas; PCV, hematocrito; WBC, leucocitos; LYM, linfocitos; MID, monocitos; GRA, neutrófilos eosinófilos basófilos; MPV, volumen plaquetario medio. ${ }^{\mathrm{a}, \mathrm{b}}$ Medias seguidas de letras distintas difieren significativamente $(\mathrm{P}<0,01)$.

PLT: platelets; PCV, hematocrit, WBC: Leukocytes; LYM: lymphocytes; MID: monocytes; GRA: neutrophils, eosinophils and, basophils. ${ }^{\text {a,b }}$ Means followed by different letters differ significantly $(\mathrm{P}<0.01)$. 
de APCs o PRP con finalidades terapéuticas en el gato, existe información sobre métodos manuales para concentrar plaquetas felinas y evaluar in vitro el efecto de sustancias agregantes y antiagregantes. En esos estudios se empleó citrato de sodio al 3,8\% como anticoagulante y se incluyeron diferentes protocolos de centrifugación que oscilaron entre 150 y $200 \mathrm{~g}$ y entre 10 y $20 \mathrm{~min}$ (Grossklaus y col 1976, Burke y col 1983a a Burke y col $1983^{b}$, Hayes y col 1989, Johnson y col 1990, Murohara y col 1996, Hayward 1999). En esas investigaciones no se presentaron datos sobre el número de plaquetas concentradas ni otra información hematológica de las características de los concentrados de plaquetas. Por esta razón, los autores consideran que la investigación presentada en este artículo representa un aporte al campo de la hematología felina con utilidad clínica en el campo de la medicina regenerativa de esa especie.

El método evaluado permitió concentrar plaquetas y disminuir significativamente los MID y GRA y porcentualmente los LYM. Es decir, la población celular concentrada fue totalmente diferente a la sanguínea basal. La correlación altamente significativa entre los niveles basales de plaquetas y PLT-APC y la correspondiente ecuación de regresión lineal indicaron que para este método el pronóstico del valor de PLT-APC estaría basado en un incremento constante de 341.163,294 más 1,650 veces el valor del número de plaquetas en sangre entera. Si bien la eficiencia de recolección de plaquetas se presenta aparentemente baja, esto podría deberse al hecho de que para efectos del presente estudio solo se tomó $1 \mathrm{~mL}$ de plasma. Según el conocimiento de los autores, los felinos representan (hasta el momento) la única especie en la que se puede predecir la concentración de plaquetas del PRP (obtenido mediante el método del tubo) en función del recuento de plaquetas de la sangre total. Esta correlación no ha sido observada en sangre de caninos (Silva y col 2011) o equinos (Argüelles y col 2006) procesada mediante el método del tubo. Sin embargo, aún queda pendiente investigar cómo se pueden correlacionar o predecir las concentraciones de los factores de crecimiento plaquetarios respecto a los recuentos celulares del PRP obtenido mediante el método del tubo en felinos.

Se ha encontrado que la calidad de los APCs o del PRP puede estar estrechamente relacionada con el anticoagulante empleado en su elaboración (Lei y col 2009). Por ejemplo, los concentrados de plaquetas elaborados con ACD son de superior calidad respecto a los concentrados plaquetarios obtenidos con citrato de sodio o heparina. Esta situación plantea una ventaja del método descrito en este artículo. Hasta ahora, los autores habían descrito dos protocolos para elaborar concentrados de plaquetas en caninos (Silva y col 2011) y equinos (Argüelles y col 2006). Se debe entender el término "concentrado de plaquetas" como plasma con una suspensión de plaquetas (viables) entre 400 a 600 mil plaquetas por microlitro (Carmona y col 2011). Sin embargo, con el método presentado aquí, se pudo obtener (desde un punto de vista arbitrario) PRP. Marx (2004) estableció que el PRP puede comprender una suspensión de un millón o más de plaquetas por microlitro de plasma.

Cuando se usan métodos semiautomatizados se puede concentrar un gran número de plaquetas. Sin embargo, el número de leucocitos se incrementa drásticamente y las plaquetas comienzan a ser activadas antes de ser empleadas clínicamente, en contraste al concentrar plaquetas con el método del tubo. Un hecho llamativo del presente estudio es que se logró obtener un PRP con un alto número de plaquetas viables con un bajo número de leucocitos. Si bien el MPV es menor en sangre entera (MPV 7,57) que en los APCs $(10,45)$, este valor plaquetario continúa dentro del valor normal reportado para la especie (Boudreaux y Ebbe 1998). Todo esto para producir un biofármaco (PRP) ideal desde el punto de vista de Marx (2004).

Los resultados de este estudio permiten concluir que la metodología empleada para concentrar plaquetas podría ser aceptable para obtener PRP en felinos como terapia regenerativa. El análisis de regresión lineal permitió predecir el valor de PLT-APC a partir de la concentración basal de PLT en sangre entera. La relevancia del método descrito en esta comunicación radica en que permite concentrar una gran cantidad de plaquetas viables con un bajo número de leucocitos, lo cual es una condición altamente deseable en un PRP (Marx 2004). Investigaciones adicionales deben ser garantizadas para conocer las características bioquímicas y ultraestructurales del PRP (activado) felino obtenido mediante esta técnica.

\section{RESUMEN}

El objetivo de este estudio fue evaluar un método manual para concentrar plaquetas de gato $y$, en consecuencia, producir concentrados autólogos de plaquetas (APCs) o plasma rico en plaquetas (PRP) con propósitos clínicos y experimentales. El PRP se obtuvo por la extracción de sangre venosa en tubos con solución ACD que fueron centrifugados a $85 \mathrm{~g}$ durante 6 minutos. Para los recuentos celulares de sangre entera y PRP, se encontraron diferencias estadísticamente significativas ( $P$ $<0,01)$ entre los valores de las plaquetas, leucocitos, granulocitos y monocitos, pero no entre los recuentos de linfocitos. Se encontró una fuerte correlación positiva $(\rho=0,734 \mathrm{P}=0,01)$ entre el número de plaquetas en sangre entera y plaquetas en el PRP. La eficiencia de recolección de plaquetas fue de $50 \%$ y la concentración de plaquetas fue de 147,7\% mayor en los PRPs, en comparación con las muestras de sangre entera. Los resultados indican que este método de centrifugación simple permite concentrar plaquetas en el gato.

\section{REFERENCIAS}

Anitua E, I Andia, B Ardanza, P Nurden, AT Nurden. 2004. Autologous platelets as a source of proteins for healing and tissue regeneration. Thromb Haemost 91, 4-15.

Anitua E, M Sánchez, G Orive, I Andia. 2007. The potential impact of the preparation rich in growth factors (PRGF) in different medical fields. Biomaterials 28, 4551-4560.

Argüelles D, JU Carmona, J Pastor, A Iborra, L Viñals, P Martínez, E Bach, M Prades. 2006. Evaluation of single and double centrifugation tube methods for concentrating equine platelets. Res Vet Sci 81, 237-245. 
Bonomi S, CR Auada, F Almeida, DC Branco, KB Braga, C Muramoto, M Amaku. 2007. Plasma rico em plaquetas combinado a hidroxiapatita na formação do calo ósseo em fraturas induzidas experimentalmente no rádio de cães. Ciênc Rural 37, 1045-1051.

Boudreaux MK, S Ebbe. 1998. Comparison of Platelet Number, Mean Platelet Volume and Platelet Mass in Five Mammalian Species. Comp Haematol Int 8, 16-20

Burke SE, G DiCola, AM Lefer. 1983a. Protection of ischemic cat myocardium by cgs-13080, a selective potent thromboxane $\mathrm{A}_{2}$ synthesis inhibitor. J Cardiovasc Pharmacol 5, 842-847.

Burke SE, AM Lefer, KC Nicolaou, GM Smith, JB Smith. 1983b. Responsiveness of platelets and coronary arteries from different species to synthetic thromboxane and prostaglandin endoperoxide analogues. Br J Pharmac 78, 287-292.

Carmona JU, M Prades. 2009. Platelet concentrates to treat musculoskeletal disease in horses. VDM Verlag Dr. Müller Aktiengesellschaft \& Co. $\mathrm{KG}$, Saarbrücken, Germany.

Carmona JU, M Prades, D Argüelles. 2009a . Autologous platelet concentrates as a treatment for soft tissue musculoskeletal lesions in horses. Arch Med Vet 41, 77-82.

Carmona JU, C López, M Prades. 2009 . Use of autologous platelet concentrates obtained by the tube method as a treatment for arthropathies in horses. Arch Med Vet 41, 175-179.

Carmona JU, C López, CE Giraldo. 2011. Use of autologous platelet concentrates as regenerative therapy for chronic diseases of the equine musculoskeletal system. Arch Med Vet 43, 1-10.

Casati MZ, BC Vasconcelos-Gurgel, PF Gonçalves, SP Pimentel, G Rochanogueira, JRFH Nociti, EA Sallum. 2007. Platelet-rich plasma does not improve bone regeneration around peri-implant bone defects-A pilot study in dogs. Int J Oral Maxillofac Surg 36, 132-136.

Chandler JC, BS Beale. 2002. Feline Orthopedics. Clin Tech Small Anim Pract 17, 190-203.

Dutra CEA, MM Pereira, R Serakides, MCF Rezende. 2008. In vivo evaluation of bioactive glass foams associated with platelet-rich plasma in bone defects. J Tissue Eng Regen Med 2, 221-227.

Grossklaus CHR, B Damerau, E Lemgo, W Vogt. 1976. Induction of Platelet Aggregation by the Complement-Derived Peptides C3a and C5a. Naunyn-Schmiedeberg's Arch Pharmacol 295, 71-76.

Hauschild G, HA Merten, A Bader, G Uhr, A Deivick, A MeyelLindenberg, M Fehr. 2005. Bioartificial bone grafting: Tarsal joint fusion in a dog using a bioartificial composite bone graft consisting of beta-tricalciumphosphate and platelet rich plasma-a case report. Vet Comp Orthop Traumatol 18, 52-54.
Hayes KG, A Pronczuk, AE Addesa, ZF Stephan. 1989. Taurine modulates platelet aggregation in cats and humans. Am J Clin Nutr 49,1211-1216.

Hayward R, B Campbell, YK Shin, R Scalia, AM Lefer. 1999. Recombinant soluble P-selectin glycoprotein ligand-1 protects against myocardial ischemic reperfusion injury in cats. Cardiovasc Res 41, 65-76

Intini G. 2009. The use of platelet-rich plasma in bone reconstruction therapy. Biomaterials 30, 4956-4966.

Johnson G, PS Tsao, D Mulloy, AM Lefer. 1990. Cardioprotective effects of acidified sodium nitrite in myocardial ischemia with reperfusion. J Pharm Exp Ther 252, 35-41.

Lei H, L Gui, R Xiao. 2009. The effect of anticoagulants on the quality and biological efficacy of platelet-rich plasma. Clin Biochem 42, 1452-1460.

Marx RE. 2004. Platelet-rich plasma: evidence to support its usage. J Oral Maxillofac Surg 62, 489-496.

Murohara T, R Scalia, AM Lefer. 1996. Lysophosphatidylcholine Promotes P-Selectin Expression in Platelets and Endothelial Cells. Possible Involvement of Protein Kinase $\mathrm{C}$ Activation and Its Inhibition by Nitric Oxide Donors. Circ Res 78, 780-789.

Monteiro SO, O M Lepage, CL Theoret. 2009. Effects of platelet-rich plasma on the repair of wounds on the distal aspect of the forelimb in horses. Am J Vet Res 70, 277-282.

Moreno L, J Marín, F Enríquez, G González, L Moreno, L Cisneros, LM Sancha. 2004. Utilización de plasma rico en plaquetas para regeneración periodontal en un perro. Rev Odont Mex 64-69.

Silva RF, CMF Rezende, FO Paes-Leme, JU Carmona. 2011. Evaluación del método del tubo para concentrar plaquetas caninas: estudio celular. Arch Med Vet 43, 95-98.

Thor A, V Franke-Stenport, CB Johansson, L Rasmusson. 2007. Early bone formation in humans bone grafts treated with platelet-rich plasma: preliminary histomorphometric results. Int J Oral Maxillofac Surg 36, 1164-1171.

Weibrich G, KGK Wilfried, B Rainer, WE Hitzler, G Hafner. 2003. The Harvest Smart PRePTM system versus the Friadent-Schütze platelet-rich plasma kit. Clin Oral Impl Res 14, 233-239.

You TM, BH Choi, J Li, JH Jung, HJ Lee, SH Lee, SM Jeong. 2007. The effect of platelet-rich plasma on bone healing around implants placed in bone defects treated With Bio-oss: a pilot study in the dog tibia. Oral Surg Oral Med Oral Pathol Oral Radiol Endod 103, 8-12. 